\title{
Recidiva del color dentario por té, café y vino. In vitro
}

\section{Dental bleaching regression caused by chromogenic beverages. In vitro}

\author{
Arévalo Pineda $\mathrm{M}^{1}$, Larrucea Verdugo $\mathrm{C}^{2}$
}

\section{RESUMEN}

Este estudio, in vitro, determinó si los dientes con clareamiento presentan mayor cambio de color en el tiempo que los no tratados, al someterse a tinción con bebidas cromógenas, café, té y vino. Se utilizaron 45 incisivos sanos de bovino conservados en suero a $37^{\circ} \mathrm{C}$. Cada espécimen se dividió en dos mitades, una sometida a clareamiento con peróxido de hidrógeno al 35\% y otra control. Se midió color con Espectrofotómetro Vita EasyShade. Se dividieron los especímenes al azar en grupos de 15 y fueron sumergidos en café, té y vino, durante 10 minutos, 20 veces, registrando color después de cada inmersión. Los datos fueron analizados con ANOVA y Test de Tukey, con 95\% de intervalo de confianza. Los resultados mostraron que, el clareamiento modifica significativamente $(p=0.05)$ el color en los tres grupos (GC=85.8 a 95.1; GT=87.4 a 97.3 y GV=90.8 a 99.3$)$, la recidiva de color se observa a lo largo de las 20 inmersiones, siendo significativa la diferencia de valores $\Delta \mathrm{E}$ iniciales $(\mathrm{GC}=18.89 ; \mathrm{GT}=22.97 ; \mathrm{GV}=56.46)$ y finales $(\mathrm{GC}=5.56 ; \mathrm{GT}=5.38 ; \mathrm{GV}=12.49)$. El grupo tratado presenta mayor descenso de unidades $\Delta \mathrm{E}$ a lo largo de las inmersiones, por lo que es el más teñido (GCcontrol=20.98-5.01; GTcontrol=17.11-3.66; GVcontrol=54.62-11.49). Las tres bebidas cromógenas causan recidiva de color en los dientes clareados, siendo el vino el que causa mayor tinción. Se concluyó que las piezas tratadas, sometidas a los tres tipos de cromógenos, tienen mayor cambio de color que las que no lo son, pero finalmente no se oscurecen más que las no tratadas.

Rev. Clin. Periodoncia Implantol. Rehabil. Oral Vol. 5(2); 57-65, 2012.

Palabras clave: Clareamiento, bebidas cromógenas, recidiva de color.

\section{ABSTRACT}

This in vitro study established if teeth treated with dental bleaching have a higher change of color over time than those that aren't treated, when subjected to three chromogenic beverages (coffee, tea and red wine). 45 healthy bovine incisors were used, maintained in $0.9 \%$ sodium chloride, at $37^{\circ} \mathrm{C}$. Every specimen was divided into 2 half; one half was subjected to dental bleaching with $35 \%$ hydrogen peroxide and the other was taken as control. The color was measured with the Vita EasyShade Spectrophotometer. The specimens were randomly divided in groups of 15, and were immersed in coffee, tea and red wine for 10 minutes, 20 times, the value being recorded after each immersion. The data was analyzed with ANOVA and Tukey test, using $95 \%$ of confidence interval. Results show that bleaching significantly modifies $(p=0.05)$ the color in the three groups $(G C=85.8$ to $95.1 ; G T=87.4$ to 97.3 y $\mathrm{GV}=90.8$ to 99.3$)$. Color relapse in the three groups was observed along the 20 immersions. Initial $\triangle E$ values $(G C=18.89 ; G T=22.97 ; G V=56.46)$ were significantly different than final $\Delta \mathrm{E}$ values $(\mathrm{GC}=5.56$; $\mathrm{GT}=5.38 ; \mathrm{GV}=12.49)$. The chromogenic beverages stained more the treated group because a higher $\Delta \mathrm{E}$ units decrease was observed in this group (GCcontrol=20.98-5.01; GTcontrol=17.11-3.66; GV control=54.62-11.49). The three chromogenic beverages produced color relapse in the bleaching teeth, being red wine the one causing more staining. It was concluded that the specimens treated subjected to three kinds of chromogenic, had higher change of color than control group, but at the end the treated group doesn't get more stained than the control group.

Rev. Clin. Periodoncia Implantol. Rehabil. Oral Vol. 5(2); 57-65, 2012.

Key words: Dental bleaching, chromogenic beverages, color relapse.

\section{INTRODUCCIÓN}

La estética es un fenómeno cultural que evoluciona con el hombre y convive paralelamente a él. En la intención de imitar la naturaleza, la estética se ha enfocado desde sus inicios a distintas áreas, es así como encontramos que la odontología estética no es un concepto actual. Desde el principio de los tiempos el ser humano ha buscado la belleza de una $u$ otra forma para agradar a los demás ${ }^{(1)}$.

Esto ha desencadenado que en la actualidad las personas no solo busquen realizarse tratamientos restaurativos estéticos, sino que además, se preocupen de complementarlos con tratamientos de clareamiento como parte importante del tratamiento dental.

El clareamiento dental es una forma efectiva para modificar la propiedad "valor" del color de las piezas dentarias, pero su efectividad puede verse dañada cuando las piezas clareadas entran en contacto con alimentos como son las bebidas cromógenas altamente consumidas en el mundo como el café, té y vino tinto, que producen pigmentaciones extrínsecas en piezas dentarias sin ningún tipo de tratamiento. Es por esto que surge la interrogante de esta investigación en la cual queremos observar si hay un mayor cambio de color en piezas clareadas que en piezas sin clareamiento al ser inmersas en este tipo de bebidas.
La sonrisa que nosotros muchas veces observamos, no nos refleja el color real de las piezas dentarias, ello producto de las pigmentaciones a las que estas diariamente se ven expuestas. El color del cromógeno es similar a la de la tinción dental. Como por ejemplo la placa bacteriana, cuyo color depende de la capacidad de absorber componentes salivales hacia el esmalte, también las tinciones del té, café, vino, metales y productos bacterianos ${ }^{(2)}$.

La odontología, con el tiempo, ha buscado técnicas alternativas a las restauraciones estéticas para tener piezas dentarías sin pigmentación y no generar un desgaste innecesario a la estructura dentaria. Esto ha llevado a optar por tratamientos como el clareamiento de piezas dentarias, técnica conservadora, sencilla, relativamente rápida y eficiente para modificar la variable "valor" del color de las piezas dentarias pigmentadas portadoras tanto de alteraciones fisiológicas, como patológicas, en piezas dentarias vitales o tratadas endodónticamente ${ }^{(3)}$.

Para llevar a cabo el clareamiento se utilizan varios tipos de productos químicos, los cuales actúan ya sea oxidando o reduciendo los agentes que causan las pigmentaciones. Las preparaciones de uso más frecuente, son las soluciones acuosas de peróxido de hidrógeno con diferentes concentraciones, el cual será utilizado en esta investigación ${ }^{(4,5)}$. 
Respecto a las concentraciones a utilizar, Gallagher et al. en $2002^{(6)}$, compararon peróxido de hidrógeno al $25 \%$ y al $38 \%$ mediante la técnica de doble ciego aleatorio. Los dos productos mostraron buenos resultados pero fue más efectiva la solución al $25 \%(3,7)$.

El clareamiento también se ha asociado con cambios en las propiedades biomecánicas de la dentina. Se dice que la exposición al peróxido de hidrógeno al $35 \%$ por 15 minutos, es suficiente para causar una disminución en la microdureza del esmalte y dentina ${ }^{(8,9,10)}$. También lo demostraron Bistey et al.(11), que observaron que la alteración en el esmalte es proporcional al tiempo de tratamiento y a la concentración de peróxido de hidrógeno. Altas concentraciones y periodos de tiempo más largos resultan en alteraciones más severas. Estos cambios en la estructura del esmalte y dentina, la hacen más susceptible a tinciones debido a la alteración en la permeabilidad y a las irregularidades dejadas en las superficies clareadas, facilitando la acumulación de pigmentos ${ }^{(8)}$.

Diferentes estudios muestran que no existe diferencia significativa entre los agentes clareadores, ya que el peróxido de carbamida y el perborato sódico, se descomponen en varios compuestos pero, el agente activo que está presente en los dos y que causa el clareamiento dental es el peróxido de hidrógeno, en relación al pronóstico y la duración que presentara este tratamiento a lo largo del tiempo, se ha observado que una vez terminado el clareamiento dental una ligera regresión del color ocurre a las dos semanas siguientes ${ }^{(12)}$.

La efectividad del clareamiento dental depende de varios factores, el factor que determina el éxito de la técnica es el color inicial de los dientes afectados, de modo que cuanto más clara sea la tinción, tanto más fácil resulta su clareamiento ${ }^{(13)}$.

La estabilidad del color puede esperarse en hasta un $90 \%$ de los pacientes después de un año del tratamiento, un $62 \%$ a 3 años y al menos $35 \%$ a los 7 años. Sin embargo, en otros estudios, se ha observado que el cambio en la coloración comienza alrededor de los primeros 6 meses de realizado el procedimiento(14).

En relación a la recidiva, no existen evidencias concretas en torno a la regresión del color experimentado por piezas dentarias sometidas a clareamiento, ni cómo ocurren; también se desconoce la predictibilidad exacta de este procedimiento, es por esto que, surge la interrogante de que si el clareamiento dental tendría alguna influencia en la recidiva del color experimentados por éstas piezas, es decir, si al realizar este procedimiento clínico, las piezas dentarias quedan más susceptibles a tener cambios de coloración ${ }^{(15)}$.

Entre los hábitos alimenticios que son más comunes en la población Latinoamericana y que están relacionados con el cambio de coloración en las piezas dentarias, están el consumo de café, té y vino tinto, por lo que es de suma importancia conocer los cromóforos existentes en la composición de cada uno de ellos.

El té, después del agua, es la bebida de mayor consumo per cápita en el mundo, con un consumo aproximado de $120 \mathrm{ml} / \mathrm{día}$, siendo el té negro la variedad mayormente consumida y producida en el mundo, con un porcentaje aproximado de entre el $76 \%$ a un $78 \%{ }^{(16)}$.

Todos los té son producidos a partir de las hojas de una planta tropical llamada Camellia sinensis, de la cual se producen las tres principales clases de té, las que se diferencian solamente por el tipo de tratamiento que reciben durante su proceso de fabricación ${ }^{(17)}$.

Después del té, el café es la bebida más comúnmente consumida en muchas partes de América Latina. Además de la cafeína y otros múltiples componentes, el café es abundante en compuestos fenólicos como ácido clorogénico, cafeico y melanoidinas, de efectos antioxidantes $\mathrm{o}$ antimutagénicos demostrados in vitro ${ }^{(18-22)}$.

El vino tinto es la bebida que le sigue en consumo al té y al café, siendo los compuestos fenólicos los responsables del color rojo del vino tinto, participan en las características sensoriales del vino y en las transformaciones durante el envejecimiento del vino. En los vinos existen diferentes familias de compuestos fenólicos: taninos, polímeros de antocianógenos y catequinas, antocianas, flavonas y ácidos fenólicos ${ }^{(23,24)}$

El café, como el té y el vino, contiene importantes antioxidantes fenólicos, tales como los ácidos clorogénico y cafeico, en algunos aspectos similares a las epicatequinas y taninos del té o las quercetinas del vino tinto, pero con diferentes estructuras químicas $\mathrm{y}$, por tanto, distintas funciones metabólicas ${ }^{(18,25)}$

Este tipo de bebidas al ser consumidas en exceso causan pigmentaciones de tipo extrínsecas en las piezas dentarias. El clareamiento dental es una forma efectiva para modificar la propiedad "valor" del color de las piezas dentarias, pero su efectividad puede verse dañada cuando las piezas clareadas entran en contacto con alimentos como son las bebidas cromógenas altamente consumidas en el mundo como el café, té y vino tinto. Es por esto que surge la interrogante de esta investigación en la cual queremos observar si hay un mayor cambio de color en piezas clareadas que en piezas sin clareamiento al ser inmersas en este tipo de bebidas, para lo que planteamos que, los dientes tratados con clareamiento dental, presentan un mayor cambio de color en el tiempo que los no tratados, al ser sometidos a tinción con tres bebidas cromógenas.

\section{MATERIAL Y MÉTODO}

Para realizar este estudio, in vitro, se seleccionaron 45 incisivos sanos de bovino, debido a que la estructura y composición química de estos es similar a la de los humanos ${ }^{(26)}$, libres de caries, sin pigmentaciones extrínsecas o intrínsecas, sin fracturas coronarias o líneas de fractura. Estos, al ser extraídos se colocaron en solución de Cloruro de Sodio al $0.9 \%$, para evitar su deshidratación, posteriormente se les realizo limpieza con ultrasonido para remover los restos orgánicos de su superficie y profilaxis con piedra pómez de grano fino en copa de goma a baja velocidad, la raíz se cortó con discos de carburundum, el contenido intracameral fue eliminado completamente con cucharetas de caries e irrigado con Cloruro de Sodio al $0.9 \%$, y conservadas nuevamente en la misma solución.

Los especímenes fueron colocados en probetas cilíndricas de resina acrílica de autocurado transparente (Figura 1), de $1.5 \mathrm{cms}$ de alto $\times 2.5 \mathrm{cms}$ de diámetro, de modo que su superficie vestibular quede libre de la resina acrílica para realizar el procedimiento.

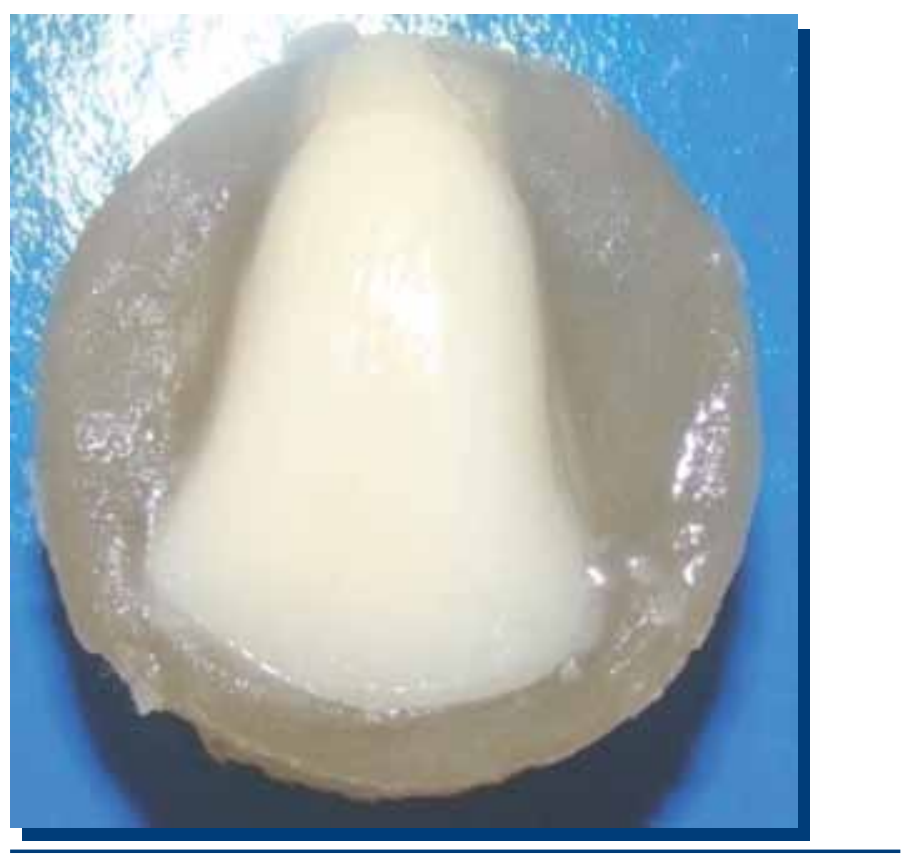

Figura 1. Pieza dentaria en probeta acrílica.

En la superficie vestibular de los especímenes se realizó una línea divisoria equivalente, con disco de carburundum, y se midió el color de cada mitad con el Espectrofotómetro Vita EasyShade (Figura 2), basado en la guía de color VITAPAN 3D-Master, de acuerdo al Sistema CIE $L^{*} a^{*} b^{*}$, el cual es una medida tridimensional de color, donde $L^{*}$ es el valor, varía de 0 (negro perfecto) a 100 (blanco perfecto), $+a^{*}$ es la medida de rojo, $y-a^{*}$, de verde. El valor $+b^{*}$ representa la medición de amarillo $\mathrm{y}-\mathrm{b}^{\star}$, de azul ${ }^{(26,27,28)}$. Los valores $\mathrm{a}^{*} \mathrm{y} \mathrm{b}^{*}$ pueden correlacionarse con tono $(\mathrm{H})$ y croma $(\mathrm{C})$, a través de una transformación matemática:

$$
\begin{aligned}
& H^{*}=\tan ^{-1}\left(b^{*} / a^{*}\right) \\
& C^{*}=\left(a^{* 2}+b^{* 2}\right)^{1 / 2}
\end{aligned}
$$




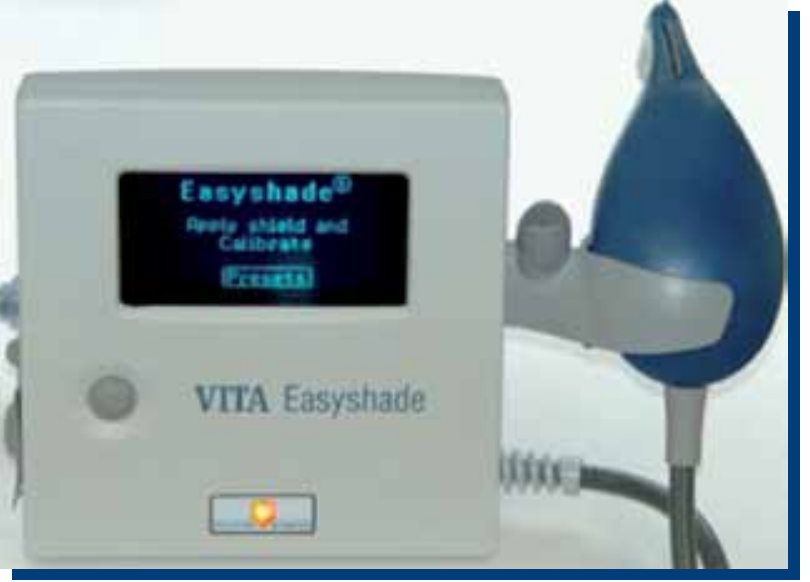

Figura 2. Espectrofotómetro EasyShade.

Los 45 especímenes fueron distribuidos al azar en 3 grupos de 15 piezas. Una mitad de la corona fue sometida a clareamiento y la otra mitad se dejó sin clareamiento, lado control. La mitad sometida a tratamiento, lo fue a través de la técnica de clareamiento externo clínico con peróxido de hidrógeno al $35 \%$ (Pola Office ${ }^{\circledR}$ ). Para separar cada mitad y evitar el escurrimiento del gel clareador hacia el lado control, se aplicó la barrera gingival flexible que viene en el set clareador (Figura 3).

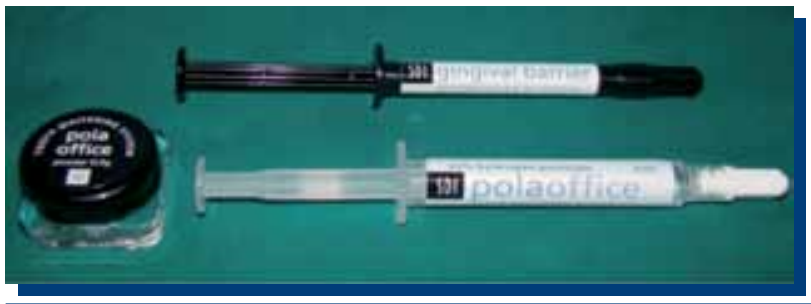

Figura 3. Tratamiento clareador Pola Office®.

Se colocó una capa gruesa de gel en la mitad de las piezas bajo tratamiento (Figura 4), se dejó durante 8 minutos, se succiono el gel con eyector y se lavó con agua (según instrucciones del fabricante), se repitió, el procedimiento 3 veces y se dejaron nuevamente en la solución de Cloruro de Sodio al $0.9 \%$ a $37^{\circ} \mathrm{C}$, en estufa Memmert.

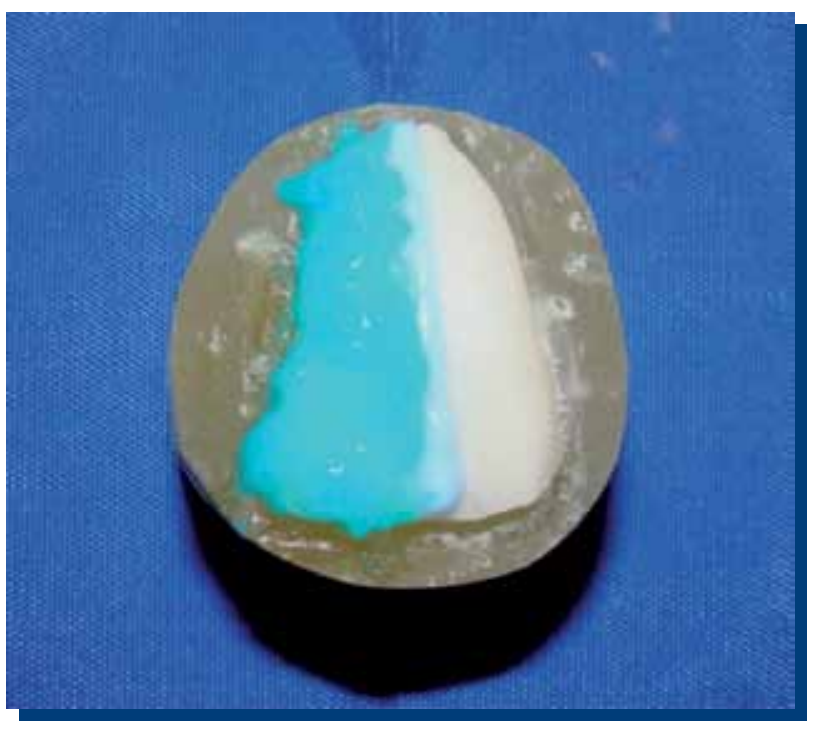

Figura 4. Especimen sometido a tratamiento clareador

Posteriormente los especímenes se dividieron en tres grupos de estudio. El primero, fue sumergido en café, usando café de grano tipo Arabica molido, preparándolo en una cafetera con 2 cucharadas de café (28 grs) en $170 \mathrm{ml}$ de agua, terminado el filtrado, se retiró de la cafetera inmediatamente y se dejó reposar hasta alcanzar $37^{\circ} \mathrm{C}$ de temperatura para sumergir los especímenes.
El segundo grupo fue sumergido en té negro marca té Club® etiqueta roja, en bolsas de $40 \mathrm{grs}$, se depositó en $200 \mathrm{ml}$ de agua a $100^{\circ} \mathrm{C}$ y se dejó reposar durante 3 minutos, una vez retirada la bolsa de té, se dejó que la infusión alcanzara los $37^{\circ} \mathrm{C}$ para la inmersión de los especímenes.

El tercer grupo fue sumergido en vino tinto tipo Cabernet Sauvignon tipo varietal año 2009 , al cual no se le realizó ningún tipo de alteración, a temperatura de inmersión de $37^{\circ}$.

Los grupos quedaron de la siguiente forma:

Grupo 1 (GC): especímenes sumergidos en café.

Grupo 2 (GT): especímenes sumergidos en té.

Grupo 3 (GV): especímenes sumergidos en vino tinto.

Todos los especímenes fueron inmersos completamente en sus respectivas soluciones, las cuales estuvieron conservadas a $37^{\circ} \mathrm{C}$ en un baño termorregulador (Figura 5 ). Se realizaron en total 20 ciclos de inmersiones de 10 minutos cada una, con un periodo de descanso de 50 minutos donde se sumergieron en Cloruro de Sodio al $0.9 \%$ a $37^{\circ} \mathrm{C}$, sin que mediara lavado previo. Después de cada 5 inmersiones se les tomo color nuevamente a ambas hemicoronas de los especímenes, obteniendo los valores $\mathrm{L}^{*} \mathrm{C}^{*} \mathrm{H}^{*}$ para cada uno.

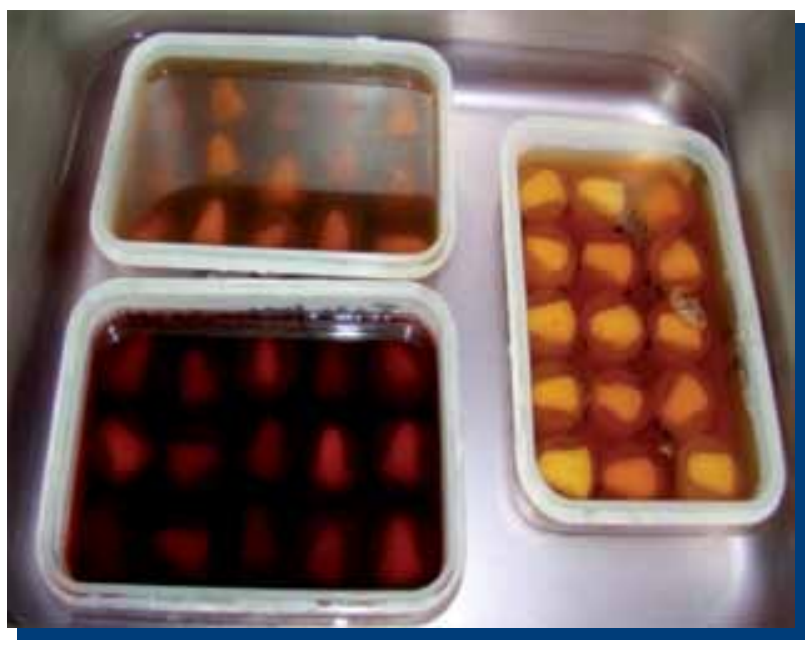

Figura 5. Especímenes inmersos en bebidas cromógenas.

El color, se determinó a través del $\Delta \mathrm{E}^{(27,29)}$, obteniendo primero el valor $\Delta$ para $\mathrm{L}^{*} \mathrm{C}^{*} \mathrm{H}^{*}$, con la siguiente fórmula:

\section{$\Delta=\mathrm{T}$ Final $-\mathrm{T}$ Inicial}

Según esta fórmula, $\Delta \mathrm{L}^{*}, \Delta \mathrm{C}^{*}$ y $\Delta \mathrm{H}^{*}$ corresponde a las diferencias respectivas en los parámetros $L^{*} C^{*} H^{*}$ de dos colores.

Luego, se obtuvieron los valores $\Delta \mathrm{E}$ calculándolos mediante la siguiente fórmula:

$$
\Delta E=\sqrt{\left(\Delta L^{*}\right)^{2}+\left(\Delta C^{*}\right)^{2}+\left(\Delta H^{*}\right)^{2}}
$$

En general, $\Delta \mathrm{E}$ representa la magnitud en la diferencia del color, pero no indica la dirección que esta diferencia sigue. Un valor $\Delta \mathrm{E}=1$ equivale al color verdadero sin diferencia entre lo medido y lo visualizado. Un valor $\Delta \mathrm{E}$ menor a 3 no es perceptible para el ojo humano ${ }^{(29)}$. Un valor $\Delta \mathrm{E}$ mayor a una o dos unidades representan cambio de color, que puede ser observado a simple vista ${ }^{(26)}$.

Se trabajó con la Media y Desviación Estándar (SD) de $\Delta \mathrm{E}$, el cual mientras mayor es su valor representa colores más claros y mientras más bajo es su valor, representa colores más oscuros. Los resultados obtenidos fueron evaluados utilizando el Software SPSS 19.0, determinándose que la Distribución de los datos es Normal, con un $95 \%$ de Intervalo de Confianza. Utilizando para esto la Prueba T para muestras independientes que utiliza el test de Tukey, y el análisis de varianza (ANOVA). 


\section{RESULTADOS}

De los 45 especímenes utilizados para este estudio, todas las mediciones se consideraron válidas, totalizando 90 superficies a las cuales se les realizo análisis de color.

Los valores del color determinados para el Grupo 1 (GC), especímenes que fueron sumergidos en café; en la primera fase experimental, clareamiento, registraron un valor promedio $L^{*} C^{*} H^{*}$ de 95.01 para el grupo tratado y de 84.95 para el grupo control, siendo esta diferencia significativa $(p=0.01)$, lo que nos indica que el clareamiento fue efectivo para modificar el color de los especímenes en estudio (Tabla 1).

Tabla 1. Valores $L^{*} C^{*} H^{*}$ iniciales para Grupo $1(G C)$

\begin{tabular}{|r|r|r|}
\hline \multicolumn{2}{|c|}{ Valores iniciales para grupos inmersos en café } \\
\hline Pieza/Inmersion & Post-clareamiento & \multicolumn{1}{|c|}{ Control } \\
\hline 1 & 92.4 & 86 \\
\hline 2 & 97 & 85.1 \\
\hline 3 & 97.7 & 89.5 \\
\hline 4 & 96.7 & 85.4 \\
\hline 5 & 91.2 & 81.1 \\
\hline 6 & 92.6 & 83.9 \\
\hline 7 & 89.7 & 88.3 \\
\hline 8 & 92.8 & 82.8 \\
\hline 9 & 98.9 & 85.1 \\
\hline 10 & 93.4 & 81.5 \\
\hline 11 & 103.9 & 80.4 \\
\hline 12 & 93.3 & 84.6 \\
\hline 13 & 100.1 & 86.1 \\
\hline 14 & 96.3 & 86.3 \\
\hline 15 & 89.1 & 88.2 \\
\hline Promedio & $\mathbf{9 5 . 0 1}$ & $\mathbf{8 4 . 9 5}$ \\
\hline
\end{tabular}

En la fase de inmersión en café, se determinaron los valores $\Delta \mathrm{E}$, de los grupos tratado y control, después de las inmersiones $1,5,10$, 15 y 20; observando la disminución de los valores $\Delta \mathrm{E}$ posterior a cada periodo de inmersión (Tabla 2). al realizar la prueba T. Anterior a la primera inmersión los dientes del grupo tratado están más claros que los del grupo control, teniendo un valor promedio $\mathrm{L}^{*} \mathrm{C}^{*} \mathrm{H}^{*}$ de 95.01 y 84.95 respectivamente, posterior a la primera inmersión, observamos al grupo tratado, con un valor de la media $\Delta \mathrm{E}$ inicial de $18.89(\mathrm{SD}=10.92)$ tiende a estar más oscuro que el grupo control que presenta un valor $\Delta \mathrm{E}$ inicial de $20.98(\mathrm{SD}=5.95)$ sin que esta diferencia sea estadísticamente significativa $(p=0.314)$; de la primera a la quinta inmersión, se observa un aparente aclaramiento de ambos grupos, esta diferencia, aunque no es significativa con la primera inmersión $(p=0.545)$, sugiere que el cromógeno en las primeras exposiciones no se adhiere al diente, permitiendo que este sea eliminado en los periodos de tiempo que los especímenes estuvieron inmersos en suero fisiológico, observamos también que el grupo tratado mantiene la tendencia a ser más oscuro que el grupo control, siendo la diferencia entre ellos no estadísticamente significativa. Después la inmersión 10 , los valores $\Delta \mathrm{E}$ mantienen la tendencia a disminuir (Tratado=21.73; Control=21.34), sin que haya diferencia estadísticamente significativa $(p=0.994)$ con los valores de la inmersión 5 (Tratado=22.74; Control=26.90). De la inmersión 10 a la 15, el oscurecimiento de los especímenes, es evidente en ambos grupos, captan más cromógeno a partir de esta inmersión y disminuyen sus valores $\Delta \mathrm{E}$, hay diferencia significativa (ptratado $=0.001$; pcontrol $=0.00$ ) con respecto a las inmersiones anteriores, sin embargo el cromógeno afecta de manera similar a ambos grupos (Tratado=10.95; Control=10.47) ya que no hay diferencias significativas entre ellos $(p=0.4075)$. Finalmente, en la inmersión 20 , se observa una tendencia de ambos grupos a oscurecerse aún más (Tratado=5.56; Control=5.01) aunque $\sin$ que exista diferencia significativa con la inmersión 15 $(p=0.213)$. Al final de las 20 inmersiones ambos grupos adquieren un valor $\Delta \mathrm{E}$ muy inferior al inicial, quedando los especímenes más oscuros que al principio. El café tiñe, inicialmente, más a los dientes clareados pero finalmente tiñe a ambos grupos de una forma similar (Gráfico 1).

Tabla 2. Valores $\Delta \mathrm{E}$ Grupo 1 (GC).

\begin{tabular}{|c|c|c|c|c|c|c|c|c|c|c|}
\hline & Café 1 Tratado & Café 1 Control & Café 5 Tratado & Café 5 Control & Café 10 Tratado & Café 10 Control & Café 15 Tratado & Café 15 Control & Café 20 Tratado & Café 20 Control \\
\hline & ${ }_{-}^{\mathrm{E}}$ & ${ }_{-}^{E}$ & $\mathrm{E}^{\mathrm{E}}$ & ${ }_{-}^{\mathrm{E}}$ & $\mathrm{E}^{\mathrm{E}}$ & $E^{E}$ & ${ }_{-} \mathrm{E}$ & E $^{\mathrm{E}}$ & ${ }_{-}^{E}$ & ${ }_{-} \mathrm{E}$ \\
\hline 1 & 1325 & 2332 & 19.79 & 2298 & 2490 & 863 & 781 & 306 & 208 & 250 \\
\hline 2 & 34,43 & 1325 & 2160 & 3458 & 21.59 & 1421 & 849 & 1000 & 429 & 717 \\
\hline 3 & 2782 & 2326 & 2239 & 2861 & 20,72 & 8.53 & 1201 & 225 & 750 & 11.7 \\
\hline 4 & 9.55 & 3649 & 2297 & 3257 & 2637 & 2733 & 19.11 & 2024 & 725 & 505 \\
\hline 5 & 12.12 & 25.74 & 4085 & 3148 & 625 & 1730 & 571 & 333 & 427 & 103 \\
\hline 6 & 3951 & 2120 & 19.57 & 2501 & 1586 & 2233 & 580 & 459 & 488 & 5.11 \\
\hline 7 & 3.00 & 1800 & 21.74 & 2454 & 781 & 1820 & 325 & 966 & 3.10 & 398 \\
\hline 8 & 1279 & 1893 & 22.74 & 2381 & 1745 & 1740 & 1203 & 737 & 10.57 & 392 \\
\hline 9 & 8.12 & 1770 & 2791 & 2438 & 2922 & 3150 & 905 & 1338 & 831 & 0.57 \\
\hline 10 & 1038 & 2725 & 19.57 & 2905 & 25.48 & 2614 & 13.7 & 1705 & 650 & 405 \\
\hline 11 & 2490 & 1968 & 2367 & 2764 & 24.54 & 3442 & 1162 & 1521 & 591 & 13.55 \\
\hline 12 & 2642 & 11.75 & 1363 & 2653 & 24.73 & 2045 & 1172 & 917 & 525 & 745 \\
\hline 13 & 2057 & 19.13 & 1920 & 2216 & 2828 & 2159 & 765 & 1621 & 650 & 317 \\
\hline 14 & 2976 & 2093 & 2090 & 2585 & 2682 & 2797 & 1291 & 730 & 541 & 222 \\
\hline 15 & 1069 & 1813 & 24.51 & 2423 & 2589 & 2403 & 2329 & 18.19 & 136 & 333 \\
\hline & & & & & & & & & & \\
\hline Mean & 18.89 & 20.98 & 274 & 26.90 & 21.3 & 2134 & 10.95 & 10.47 & 5.56 & 5.01 \\
\hline SD & 10.92 & 5.95 & 5.91 & 3.69 & 7.03 & 7.58 & 5.19 & 5.93 & 241 & 3.65 \\
\hline
\end{tabular}

Al analizar los resultados de $\Delta \mathrm{E}$ de los grupos tratado y control inmersos en café, en los tiempos de inmersión mencionados, observamos una mayor tendencia a la recidiva del color en los dientes tratados sin que esta diferencia alcance valores estadísticamente significativos, obtenidos 


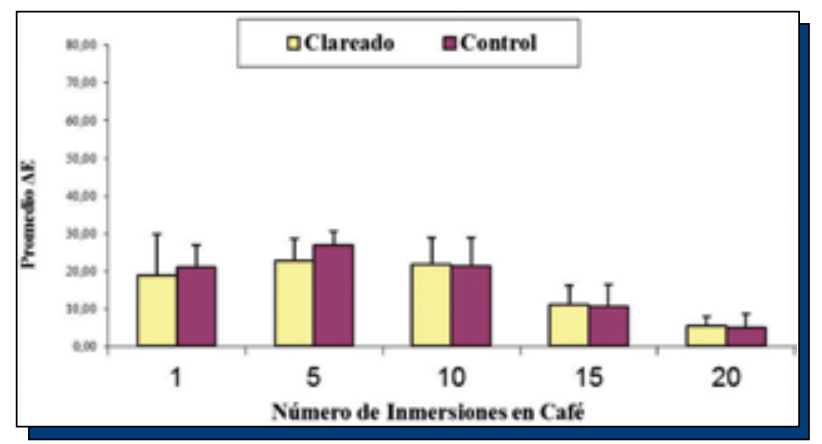

Gráfico 1. Diferencia en el cambio de color entre los grupos tratado y control, inmersos en café por tiempo de inmersión.

Los valores del color determinados para el Grupo 2 (GT), especímenes que fueron sumergidos en té, en la primera fase experimental, clareamiento, registraron un valor promedio $\mathrm{L}^{*} \mathrm{C}^{*} \mathrm{H}^{*}$ de 97.29 para el grupo tratado y de 88.73 para el grupo control, siendo esta diferencia significativa $(p=0.02)$, lo que nos indica que este tratamiento, al igual que en él Grupo 1, fue efectivo para modificar el color de los especímenes en estudio (Tabla 3).

Tabla 3. Valores iniciales para Grupo 2 (GT).

\begin{tabular}{|r|r|r|}
\hline \multicolumn{2}{|c|}{ Valores iniciales para grupos inmersos en té } \\
\hline Pieza/Inmersion & Post-clareamiento & \multicolumn{1}{c|}{ Control } \\
\hline 1 & 96.7 & 93.3 \\
\hline 2 & 96.4 & 97.5 \\
\hline 3 & 96.9 & 90 \\
\hline 4 & 102.3 & 89 \\
\hline 5 & 96.9 & 79.6 \\
\hline 6 & 98.6 & 91.1 \\
\hline 7 & 97.9 & 85.7 \\
\hline 8 & 82.6 & 86 \\
\hline 9 & 89 & 88.8 \\
\hline 10 & 92.7 & 89.6 \\
\hline 11 & 96.9 & 85.1 \\
\hline 12 & 94.2 & 90.7 \\
\hline 13 & 105.8 & 83.7 \\
\hline 14 & 109.1 & 89.5 \\
\hline 15 & 103.4 & 91.4 \\
\hline Promedio & $\mathbf{9 7 . 2 9}$ & $\mathbf{8 8 . 7 3}$ \\
\hline SD & $\mathbf{6 . 5 1}$ & $\mathbf{4 . 2 8}$ \\
\hline
\end{tabular}

En la fase de inmersión en té, se determinaron los valores $\Delta \mathrm{E}$, de los grupos tratado y control, después de las inmersiones 1, 5, 10, 15 y 20 ; observando la disminución de los valores $\Delta \mathrm{E}$ posteriores a cada periodo de inmersión (Tabla 4).
Al analizar los resultados de $\Delta E$ de los grupos tratado y control inmersos en té, en los tiempos de inmersión mencionados, observamos una mayor tendencia a la recidiva del color en los dientes tratados sin que esta diferencia alcance valores estadísticamente significativos, obtenidos al realizar la prueba T. Anterior a la primera inmersión los dientes del grupo tratado están más claros que los del grupo control, teniendo un valor promedio $\mathrm{L}^{*} \mathrm{C}^{*} \mathrm{H}^{*}$ de 97.29 y 88.75 respectivamente, posterior a la primera inmersión, observamos al grupo tratado, con un valor de la media $\Delta \mathrm{E}$ inicial de $22.97(\mathrm{SD}=12.34)$ tiende a estar más claro que el grupo control que presenta un valor $\Delta \mathrm{E}$ inicial de $17.11(\mathrm{SD}=4.70)$ sin que esta diferencia sea estadísticamente significativa $(p=0.85)$; de la primera a la quinta inmersión, se observa un aparente aclaramiento de ambos grupos, esta diferencia, aunque no es significativa con la primera inmersión $(p=0.318)$, sugiere que el cromógeno en las primeras exposiciones no se adhiere suficientemente al diente, similar a lo acontecido con el Grupo 1 , permitiendo que este sea eliminado en los periodos de tiempo que los especímenes estuvieron inmersos en suero fisiológico, observamos también que el grupo tratado mantiene la tendencia a ser más claro que el grupo control, no siendo la diferencia entre ellos estadísticamente significativa $(p=0.2345)$. Después de la inmersión 10 , el oscurecimientos de los especímenes, es evidente en ambos grupos, captan más cromógeno a partir de esta inmersión y disminuyen los valores $\Delta \mathrm{E}$, hay diferencia estadísticamente significativa $(p=0.00)$ con respecto a las inmersiones anteriores, sin embargo el cromógeno afecta más al grupo control (Tratado $=9.56$; Control $=8.08$ ) ya que hay diferencias significativas con el grupo tratado $(p=0.05)$. De la inmersión 10 a la 15 , no hay diferencia significativa ( $p=0.461)$ en el oscurecimiento de los especímenes, aunque los valores de ambos grupos disminuyen (Tratado=5.49; Control=4.10). Finalmente a la inmersión 20, se observa una tendencia de ambos grupos a oscurecerse aún más (Tratado $=5.38$; Control=3.66) aunque sin que exista diferencia significativa con la inmersión $15(p=1.00)$, pero si se observa diferencia significativa entre grupos $(p=0.04)$, entre los cuales el grupo control es el que termina más oscuro, con valores $\Delta \mathrm{E}$ menores. Al final de las 20 inmersiones, ambos grupos presentan un valor $\Delta \mathrm{E}$ muy inferior al valor inicial, quedando los especímenes más oscuros que al principio. Si consideramos que el grupo tratado originalmente era más claro que el control, y termino con valores similares, observamos una mayor diferencia de color, con una clara tendencia a oscurecerse por parte del grupo tratado (Gráfico 2).

Tabla 4. Valores $\Delta \mathrm{E}$ para Grupo 2 (GT).

\begin{tabular}{|c|c|c|c|c|c|c|c|c|c|c|}
\hline & Té 1 Tratado & Té 1 Control & Té 5 Tratado & Té 5 Control & Té 10 Tratado & Té 10 Control & Té 15 Tratado & Té 15 Control & Té 20 Tratado & Té 20 Control \\
\hline & $E_{-}^{E}$ & - $^{E}$ & E & $I^{E}$ & $E^{E}$ & \begin{tabular}{|c|}
$E$ \\
\end{tabular} & \begin{tabular}{|c|}
$E$ \\
\end{tabular} & $E^{E}$ & \begin{tabular}{|c|}
$\varepsilon$ \\
\end{tabular} & $E_{-}^{E}$ \\
\hline & 10.02 & 5.38 & 25.64 & 26.93 & 9.31 & 8.57 & 2.34 & 1.30 & 4.12 & 4.08 \\
\hline & 18.42 & 18.85 & \begin{tabular}{ll|l|}
19.71 \\
\end{tabular} & 22.57 & 15.43 & 9.88 & 5.42 & 4.70 & 3.42 & 6.81 \\
\hline & 23.28 & 20.09 & 24.19 & 31.92 & 11.88 & 9.72 & 1.54 & 1.10 & 4.02 & 1.62 \\
\hline & 12.73 & 19.72 & 30.37 & 20.84 & 4.68 & 6.36 & 2.24 & 3.47 & 6.35 & 2.28 \\
\hline & 20.84 & 19.00 & 27.83 & 32.05 & 7.02 & 7.50 & 2.42 & 4.86 & 4.30 & 0.64 \\
\hline & 8.72 & 20.72 & 27.46 & 24.91 & 11.05 & 7.11 & 3.12 & 7.85 & 8.93 & 7.01 \\
\hline & 32.22 & 22.11 & 24.96 & 22.99 & 7.18 & 8.74 & 1.70 & 3.17 & 4.31 & 2.30 \\
\hline & 12.11 & 13.96 & 19.12 & 24.23 & 10.43 & 10.97 & 5.22 & 7.04 & 6.67 & 3.45 \\
\hline & 14.13 & 13.00 & 26.33 & 27.50 & 10.83 & 6.34 & 4.75 & 2.91 & 0.89 & 139 \\
\hline 10 & 26.43 & 14.57 & 30.41 & 22.56 & 730 & 78.86 & 4.45 & 3.23 & 2.05 & 1.05 \\
\hline 11 & 17.78 & 18.12 & 24.31 & 27.64 & 10.56 & 875 & 5.97 & 3.20 & 3.20 & 7.86 \\
\hline 12 & 22.98 & 20.38 & 25.09 & 24.90 & 7.55 & 581 & 14,44 & 4.97 & 11.07 & 7.38 \\
\hline 13 & 35.75 & 20.79 & 27.24 & 32.26 & 8.52 & 12.89 & 7.61 & 1.42 & 6.51 & 2.64 \\
\hline 14 & 34.11 & 19.67 & 44.74 & 26.59 & 12.31 & 4.63 & 10.83 & 4.25 & 4.80 & 3.19 \\
\hline 15 & 54.95 & 10.26 & 37.29 & 2585 & 9.31 & 6.00 & 10.28 & 8.01 & 9.99 & 3.17 \\
\hline & & & & & & & & & & \\
\hline Mean & 2297 & 17.11 & 27.65 & 26.25 & 9.56 & 8.08 & 5.49 & 4.10 & 5.38 & 3.66 \\
\hline & 1234 & 4.70 & 6.43 & 3.60 & 2.56 & 219 & 3.81 & 2.20 & 288 & 2.44 \\
\hline
\end{tabular}




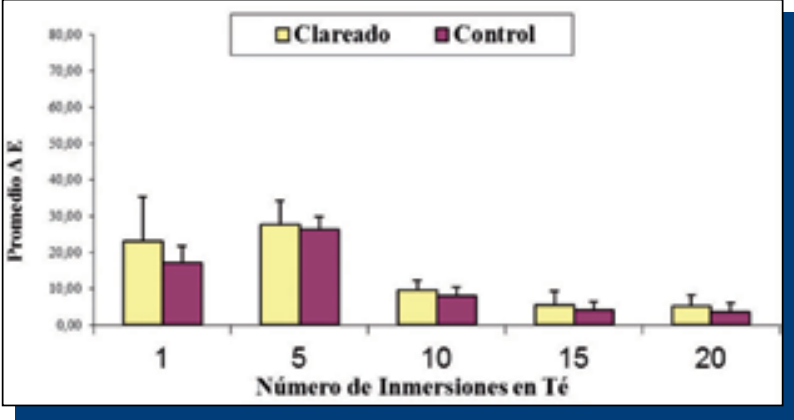

Gráfico 2. Diferencia en el cambio de color entre los grupos tratado y control, inmersos en té por tiempo de inmersión.

Los valores del color determinados para el Grupo 3 (GV), especímenes que fueron sumergidos en vino, en la primera fase experimental, clareamiento, registraron un valor promedio $\mathrm{L}^{*} \mathrm{C}^{*} \mathrm{H}^{*}$ de 99.38 para el grupo tratado y de 87.80 para el grupo control, siendo esta diferencia significativa ( $p=0.02$ ), lo que nos indica, al igual que los grupos anteriores, que este tratamiento fue efectivo para modificar el color de los especímenes en estudio (Tabla 5).

Tabla 5. Valores iniciales para Grupo 3 (GV).

\begin{tabular}{|r|r|r|}
\hline \multicolumn{3}{|c|}{ Valores iniciales para grupos inmersos en vino } \\
\hline Pieza/Inmersion & Post-clareamiento & \multicolumn{1}{c|}{ Control } \\
\hline 1 & 99.1 & 91.3 \\
\hline 2 & 105.3 & 88.5 \\
\hline 3 & 95.4 & 85.7 \\
\hline 4 & 106.2 & 84.4 \\
\hline 5 & 101.5 & 89.9 \\
\hline 6 & 99.8 & 91 \\
\hline 7 & 97.4 & 86.8 \\
\hline 8 & 96.6 & 84.6 \\
\hline 9 & 99.2 & 88.5 \\
\hline 10 & 101.6 & 85.8 \\
\hline 11 & 95.6 & 85.1 \\
\hline 12 & 93.7 & 89.8 \\
\hline 13 & 97.6 & 89.7 \\
\hline 14 & 100.8 & 85.8 \\
\hline Promedio & 100.9 & 90.1 \\
\hline SD & $\mathbf{9 9 . 3 8}$ & $\mathbf{8 7 . 8 0}$ \\
\hline
\end{tabular}

En la fase de inmersión en vino, se determinaron los valores $\Delta \mathrm{E}$, de los grupos tratado y control, después de las inmersiones $1,5,10$, 15 y 20 ; observando la disminución de los valores $\Delta \mathrm{E}$ posteriores a cada periodo de inmersión (Tabla 6).

Tabla 6. Valores $\Delta \mathrm{E}$ para Grupo $3(\mathrm{GV})$.
Al analizar los resultados de $\Delta \mathrm{E}$ de los grupos tratado y control inmersos en vino, en los tiempos de inmersión mencionados, observamos una mayor tendencia a la recidiva del color en los dientes tratados sin que esta diferencia alcance valores estadísticamente significativos, obtenidos al realizar la prueba T. Anterior a la primera inmersión los dientes del grupo tratado están más claros que los del grupo control teniendo un valor promedio $\mathrm{L}^{*} \mathrm{C}^{*} \mathrm{H}^{*}$ de 99.38 y 87.80 respectivamente, posterior a la primera inmersión, observamos que al grupo tratado, con un valor de la media $\Delta \mathrm{E}$ inicial de $56.46(\mathrm{SD}=20.09)$ tiende a estar más claro que el grupo control que presenta un valor $\Delta \mathrm{E}$ inicial de 54.62 $(S D=13.42)$ sin que esta diferencia sea estadísticamente significativa $(p=0.385)$; de la primera a la quinta inmersión, se observa disminución significativa $(p=0.00)$ de los valores $\Delta \mathrm{E}$ en ambos grupos (Tratado=33.13; Control=29.69); se mantiene el grupo tratado significativamente $(p=0.05)$ más claro que el grupo control. Después de la inmersión 10 , los valores $\Delta \mathrm{E}$ mantienen la tendencia a disminuir $(T r a t a d o=20.03$; Control=21.86), mostrando diferencia estadísticamente significativa $(p=0.004)$ con los valores de la inmersión 5 (Tratado=33.13; Control=29.69); en esta inmersión podemos observar que hay una disminución significativa $(p=0.05)$ entre grupos, en la cual el grupo tratado es más oscuro que el grupo control, captando más cromógeno durante este periodo. De la inmersión 10 a la 15, el oscurecimiento de los especímenes, es evidente en ambos grupos, captan más cromógeno a partir de esta inmersión y disminuyen sus valores $\Delta \mathrm{E}$, hay diferencia significativa (ptratado $=0.05$; pcontrol $=0.00$ ) con respecto a las inmersiones anteriores, sin embargo el cromógeno afecta de manera similar a ambos grupos ya que no hay diferencias significativas entre ellos $(p=0.311)$. Finalmente en la inmersión 20, se observa una tendencia de ambos grupos a oscurecerse aún más (Tratado=12.49; Control=11.49) aunque sin que exista diferencia significativa con la inmersión $15(p=0.963)$. Al final de las 20 inmersiones ambos grupos adquieren un valor $\Delta \mathrm{E}$ muy inferior al inicial, quedando los especímenes más oscuros que al principio. Si consideramos que el grupo tratado empezó más claro que el control y termino con valores similares, observamos una mayor tendencia a oscurecerse por parte del grupo tratado (Gráfico 3).

\begin{tabular}{|c|c|c|c|c|c|c|c|c|c|c|}
\hline & Vino 1 Trabado & Vino 1 Control & Vino 5 Tratado & Vino 5 Control & Vino 10 Tratado & Vino 10 Control & Vino 15 Tratado & Vino 15 Control & Vino 20 Tratado & Vino 20 Control \\
\hline & $E^{E}$ & $\mathrm{E}^{\mathrm{E}}$ & \begin{tabular}{|c|}
$E$ \\
\end{tabular} & ${ }_{-1}^{E}$ & E & $\mathrm{f}^{\mathrm{E}}$ & I $^{E}$ & $t_{-}^{E}$ & ${ }_{-}^{\mathrm{E}}$ & ${ }_{-}^{E}$ \\
\hline 1 & 5571 & 3956 & 3031 & 1720 & 1602 & 2633 & 248 & 757 & 1158 & 1266 \\
\hline 2 & 4835 & 39.84 & $35 / 3$ & $24 \sqrt{3}$ & 187 & 24,53 & 629 & 7.15 & 426 & 1299 \\
\hline 3 & 6.18 & 2281 & 2195 & 3385 & 1830 & 2190 & 860 & 1032 & 1486 & 1709 \\
\hline 4 & 5015 & 5816 & 3754 & 3971 & 1982 & 1729 & 1102 & 1008 & 1588 & 1056 \\
\hline 5 & 2879 & 55.2 & 3279 & 32.2 & 1756 & 2292 & 1136 & 235 & 1091 & 1083 \\
\hline 6 & 2197 & 6301 & $29 \pi$ & 2907 & 2600 & 2240 & 7664 & 733 & $16 \pi$ & 1621 \\
\hline 7 & 799 & 6133 & 4097 & 2891 & 229 & 2285 & 7.2 & 759 & $11 / 3$ & 1386 \\
\hline 8 & 8.19 & 60.12 & 3452 & 3117 & 1529 & 1685 & 1279 & 1100 & 1623 & 200 \\
\hline 9] & 3108 & 7053 & 2871 & 3564 & 2007 & 1887 & 1257 & 782 & $10 / 2$ & 1059 \\
\hline 10 & 3941 & 5797 & 3905 & 2682 & 2411 & 2107 & $10: 38$ & $111 \%$ & 7.74 & 443 \\
\hline 11 & 675 & 3835 & 2791 & 1981 & 23.18 & 2197 & 11.78 & 858 & 1274 & 886 \\
\hline 12 & 7253 & 6552 & 2530 & 296 & 1733 & 2127 & 7.14 & $9 B 5$ & 1333 & 623 \\
\hline $\mathrm{B}$ & 74.78 & 6081 & 3422 & 3301 & $22 \pi$ & $23 \mathrm{Ag}$ & $11 \Omega$ & 1022 & 5.16 & 507 \\
\hline 14 & 7847 & 5654 & 3847 & 2780 & 229 & 22.16 & 1588 & 1072 & 1993 & 1412 \\
\hline 15 & 4784 & 6925 & 3860 & 3547 & 149 & 2383 & 1384 & 1181 & 16008 & 1584 \\
\hline Mean & 5646 & $5 . Q 2$ & 33.13 & 29.69 & 20.03 & 2185 & 10.12 & 9.63 & 1249 & 1149 \\
\hline SD & 20.09 & 13.42 & 5.63 & 5.97 & 3.45 & 257 & 3.43 & 179 & 4.40 & 4.02 \\
\hline
\end{tabular}




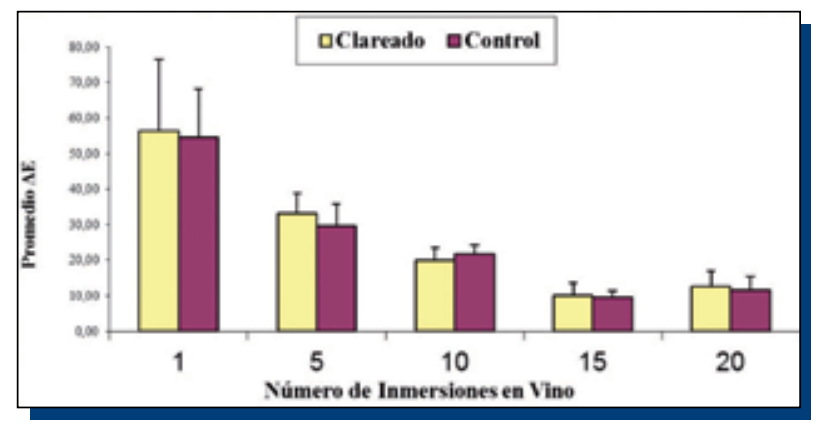

Gráfico 3. Diferencia en el cambio de color entre los grupos tratado y control, inmersos en vino por tiempo de inmersión.

Los resultados obtenidos al analizar los Gráficos 1-3 sugieren que los especímenes, en general, alcanzan su peak de mayor tinción alrededor de las inmersiones 10-15, no habiendo cambio de color significativo después de estas inmersiones, en los grupos tratados como controles.

Para analizar los resultados de $\Delta \mathrm{E}$ obtenidos con el análisis de varianza (ANOVA) para la comparación entre cromógenos del grupo tratado, debemos tener en cuenta los valores $L^{*} C^{*} H^{*}$ iniciales de cada grupo $(\mathrm{GC}=95.01 ; \mathrm{GT}=97.29$ y $\mathrm{GV}=99.38)$ en los cuales observamos que GV inicia con valores más altos que los otros grupos. En la primera inmersión, el grupo más oscuro es GC mostrando diferencia estadísticamente significativa con GV $(p=0.00)$, que sigue siendo el grupo más claro, pero no muestra diferencia significativa con GT $(p=0.739)$. En la quinta inmersión, GC sigue siendo el que muestra menores valores $\Delta E$, conservando la diferencia significativa con $G V(p=0.00)$; sin embargo, podemos observar que, durante esta inmersión GV, tiene mayor descenso en sus valores, mientras que GC y GT, presentan una tendencia a perder cromógeno. En la inmersión 10 encontramos una tendencia de GT a oscurecerse más que los otros grupos, presentando diferencia significativa con estos $(p=0.00), G C$ se mantiene con valores similares a los de la inmersión 5, mientras que GV sigue con la tendencia a disminuir sus valores, pero no muestra diferencias significativas con $\mathrm{GC}(\mathrm{p}=0.599)$. Durante la inmersión 15 los tres grupos disminuyen sus valores, mostrando el mayor descenso GV; GT sigue siendo el grupo más oscuro mostrando diferencias significativas con GC $(p=0.003)$ y GV $(p=0.01)$. A la inmersión $20, G V$ mantiene valores similares a los de la inmersión 15, pudiendo concluir que después de esta inmersión, este grupo tiende a presentar saturación del color, ya que aparentemente, no absorbe más cromógeno y sus valores se estabilizan. GC, durante la última inmersión, presento disminución en el color llegando a valores similares a los de GT, no habiendo diferencia significativa entre ellos $(p=0.988)$. Si consideramos que $G V$, después de la primera inmersión, tenía valores más altos que los otros grupos y termino con valores solo un poco mayores que estos, podemos decir que el vino es el cromógeno que más afecta a las piezas con clareamiento (Gráfico 4).

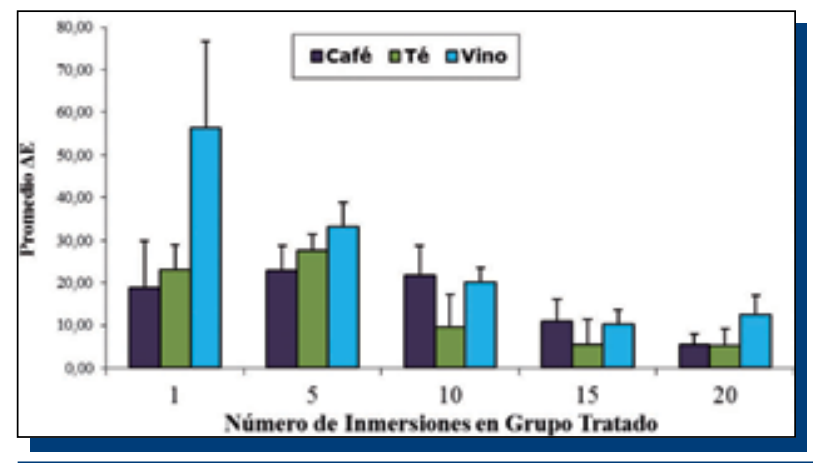

Gráfico 4. Comparación entre cromógenos por inmersión.

Para analizar los resultados de $\Delta \mathrm{E}$ obtenidos con el análisis de varianza (ANOVA) para la comparación entre cromógenos del grupo control, debemos tener en cuenta los valores $\mathrm{L}^{*} \mathrm{C}^{*} \mathrm{H}^{*}$ iniciales de cada grupo $(\mathrm{GC}=84.95 ; \mathrm{GT}=88.73$ y $\mathrm{GV}=87.80)$ en los cuales observamos que GT y GV inician con valores más altos que GC. A la primera inmersión, el grupo que muestra el mayor descenso es GT, siendo el grupo más oscuro en esta inmersión y mostrando diferencia estadísticamente significativa con GV $(p=0.00)$, pero no con $G C(p=0.464)$. En la quinta inmersión, GT sigue siendo el que muestra menores valores $\Delta E$, conservando la diferencia significativa con $G V(p=0.00)$; sin embargo, podemos observar también, que durante esta inmersión GV, tuvo un mayor descenso en sus valores, mientras que GC y GT, presentaron una tendencia a perder cromógeno. En la inmersión 10 encontramos una tendencia de GT a oscurecerse más que los otros grupos, presentando diferencia significativa con estos $(p=0.00), G C$ se mantiene con valores similares a los de la inmersión 5 , mientras que GV sigue con la tendencia a disminuir sus valores, pero no muestra diferencias significativas con $G C(p=0.953)$. Durante la inmersión 15 los tres grupos disminuyen sus valores $(G C=10.47$; $\mathrm{GT}=4.10 ; \mathrm{GV}=9.63$ ), mostrando el mayor descenso GV; GT sigue siendo el grupo más oscuro mostrando diferencias significativas con $G C(p=0.00)$ y GV $(p=0.00)$. A la inmersión 20 , GV mantiene valores similares a los de la inmersión 15, pudiendo concluir que después de esta inmersión, este grupo presenta saturación del color, ya que no absorbe más cromógeno y sus valores se estabilizan. GC y GT presentaron disminución en el color, siendo GT el grupo con los valores más bajos. Si consideramos que GV, después de la primera inmersión, tenía valores más altos que los otros grupos y termino con valores solo un poco mayores que estos, podemos decir que el vino es el cromógeno que más afecta a los dientes aun sin ser previamente clareados (Gráfico 5).

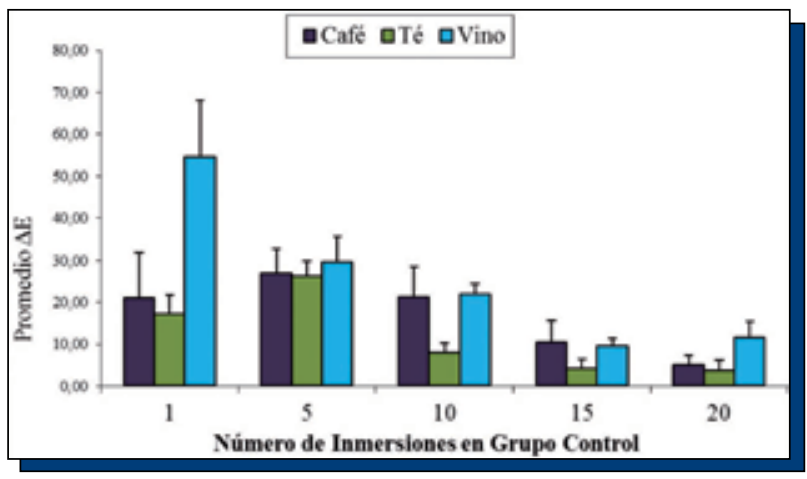

Gráfico 5. Comparación entre cromógenos por inmersión.

Al analizar los Gráficos 4 y 5 observamos que, GV mantiene descensos bruscos de valores $\Delta \mathrm{E}$ a lo largo de las 20 inmersiones, a diferencia de GC y GT que presentan descensos más paulatinos; lo que sugiere que el cromógeno que provoca mayor tinción a lo largo del tiempo, es el vino.

\section{DISCUSIÓN}

En este estudio, todos los dientes expuestos a clareamiento y posteriormente inmersos en diferentes tipos de cromógeno, presentaron un oscurecimiento más brusco que los dientes no clareados, ya que al término de las inmersiones, presentaron valores de color similares, siendo que, los dientes tratados registraron inicialmente, un color más claro. Por lo que frente a la hipótesis planteada podemos afirmar que los dientes tratados con clareamiento dental, presentan una mayor recidiva de color en el tiempo que los no tratados, al ser sometidos a tinción con tres bebidas cromógenas.

Entre los resultados obtenidos para los grupos tratado y control inmersos en café, en los tiempos de inmersión mencionados, observamos una mayor tendencia a la recidiva del color en los dientes tratados, al inicio de las inmersiones ${ }^{(1)}$, ambos grupos se oscurecen, pero el grupo tratado no solo, tiende a estar más oscuro que el grupo control aunque sin diferencias estadísticamente significativas entre ambos, si no que debemos considerar que el grupo tratado inicialmente es más claro que el control; las diferencias entre grupo disminuyen a partir de la inmersión 10 hacia las siguientes inmersiones, en las cuales ambos grupos presentan valores $\Delta \mathrm{E}$ similares. En la inmersión 5 se observa un aparente aclaramiento de ambos grupos, esta diferencia, aunque no es significativa con la primera inmersión, sugiere que el cromógeno en las primeras exposiciones no se adhiere al diente, permitiendo que este sea eliminado en los periodos de tiempo que los especímenes estuvieron inmersos en suero fisiológico. La mayor disminución de color se da entre 
la inmersión 10 y 15, en la que ambos grupos captan más cromógeno y se saturan de color disminuyendo sus valores $\Delta \mathrm{E}$ y no encontrando diferencia significativa entre estas inmersiones con la última inmersión. Al final de las inmersiones, los grupos presentan valores significativamente inferiores a los iniciales, lo que nos indica que los especímenes se oscurecieron a lo largo de estas, ya que, con el tiempo perdieron unidades $\Delta \mathrm{E}$. El café, inicialmente, tiñe más a los dientes clareados pero finalmente tiñe a ambos grupos de una forma similar.

Nuestros resultados son similares a los obtenidos por Ghavamnasiri et al. ${ }^{(26)}$, quienes concluyeron que el grupo tratado con clareamiento es significativamente más teñido que el grupo control, después de someter especímenes bovinos a clareamiento durante 2 semanas, y sumergirlos en café por 30 minutos todos los días durante 3 semanas.

En los grupos tratado y control inmersos en té, observamos, al igual que con el café, una mayor tendencia de los dientes tratados a ser más oscuros que los no tratados, sin que esta diferencia alcance valores estadísticamente significativos. Durante las primeras inmersiones ${ }^{(1)}$ observamos que el grupo tratado presenta valores más claros que el grupo control, no estadísticamente significativos, y esto se mantiene hacia la inmersión 5 , en la cual podemos observar un aparente aclaramiento de ambos grupos, al igual que acontece en el Grupo 1, sin embargo, esta diferencia de valores con la inmersión 1 no es estadísticamente significativa. A lo largo de las siguientes inmersiones, el grupo control presenta valores significativamente más bajos que el grupo tratado, sin embargo, si consideramos que el grupo tratado empezó más claro que el control y termino con valores similares, observamos una mayor tendencia a oscurecerse por parte del grupo tratado, que tiene mayor disminución de valores al comparar el color al inicio de las inmersiones con el color final $(\mathrm{T} 1=22.97 ; \mathrm{T} 20=5.38 ; \mathrm{C} 1=17.11 ; \mathrm{C} 20=3.66)$. Al finalizar las inmersiones, ambos grupos se muestran más oscuros, ya que presentan valores estadísticamente inferiores a los valores iniciales.

Leard et al.(30), en su estudio, compararon cromógenos, dentro de los cuales estaban el café y el té. Evaluaron cuál cromógeno provocaba mayor tinción, se realizó con dientes humanos, inmersos durante 15 ciclos de 10 minutos. Después de los análisis concluyeron que el té es más propenso a teñir los dientes que el café. En nuestro estudio, el té, tiende a teñir los dientes más rápido que el café, ya que el grupo inmerso en café, disminuye sus valores drásticamente a partir de la inmersión 15, en cambio el té lo hace a partir de la inmersión 10.

Con respecto a los grupos tratado y control inmersos en vino, en los tiempos de inmersión mencionados, observamos una mayor tendencia de los dientes tratados a oscurecerse, sin que esta diferencia alcance valores estadísticamente significativos. En la primera inmersión podemos observar que el grupo tratado tiende a conservar colores más claros que el control, sin que esta diferencia sea significativa. A lo largo de las inmersiones se observan disminuciones significativas en los valores, mostrando que en la inmersión 10 el grupo tratado llega a ser significativamente más oscuro que el grupo control. Los grupos estabilizan su color en la inmersión 15, no habiendo cambios significativos con la inmersión 20 , lo que sugiere que no se muestran más cambios en la luminosidad y saturación del color en los dientes a partir de esta inmersión. Al final de las inmersiones los grupos presentan un valor $\Delta \mathrm{E}$ muy inferior al valor inicial, quedando los dientes más oscuros que al principio. Si consideramos que el grupo tratado empezó más claro que el control y termino con valores similares, observamos una mayor tendencia a oscurecerse por parte del grupo tratado.

Berger et al. ${ }^{(8)}$, en su estudio concluyeron, al igual que en este estudio, que el grupo control fue más resistente a la tinción. Ellos observaron que los grupos inmersos en vino inmediatamente después del clareamiento y a las 24 horas de este, tuvieron un cambio de color cuatro veces mayor que el control. Para esto utilizaron dientes de bovino, los cuales fueron sometidos a clareamiento con peróxido de hidrógeno al
$35 \%$ durante 20 minutos y posteriormente fueron inmersos en vino tinto, inmediatamente después del tratamiento, 24 horas y 1 semana después. Al comparar cromógenos tenemos que, en el grupo tratado como en el control, el café es el cromógeno que inicialmente causa mayor oscurecimiento de los dientes, sin embargo, a lo largo de las inmersiones tiene un descenso más paulatino en sus valores. El té, por el contrario, inicialmente presenta menor descenso en el valor del color, pero, presenta descensos más bruscos a lo largo de las inmersiones, presentando así, los menores valores observados (tratado=5.38; control=3.66). Con respecto al vino, podemos observar que es el grupo que presenta mayores descensos de valor a través del tiempo, a pesar de ser el que termina con los valores más altos (tratado=12.4; control=11.49) debemos considerar que es también el grupo que comienza con los valores más altos, por lo tanto es el que presenta una mayor disminución de unidades $\Delta \mathrm{E}$, lo que significa que fueron los dientes que quedaron más oscuros al finalizar las inmersiones.

Los datos obtenidos en este estudio, con respecto a las bebidas cromógenas, son similares a los obtenidos por Toksoy et al.(28), quienes sometieron 4 marcas de resina compuesta a 8 diferentes bebidas cromógenas, entre las que se incluían vino tinto y café. Sus resultados mostraron que al finalizar 24 horas de inmersión, el cromógeno que permitió mayor tinción de las resinas, fue el vino.

Catelan et al. ${ }^{(27)}$, obtuvieron resultados similares a los de Toksoy et al. ${ }^{(28)}$, ya que sometieron 2 tipos de resina compuesta a diferentes bebidas cromógenas, las que incluían vino tinto, el cual, al término de las 4 semanas de inmersión de los especímenes, fue el cromógeno que mostro mayor tinción de las resinas.

Los resultados obtenidos en esta investigación, al igual que los estudios anteriores, nos sugieren que, los tres cromógenos utilizados provocan tinción en los dientes, siendo el vino el que mayor tinción provoca, y que el clareamiento predispone a un oscurecimiento inicial en menor tiempo.

\section{CONCLUSIONES}

Se confirma la hipótesis, los dientes tratados con clareamiento dental, presentan un mayor cambio de color en el tiempo que los no tratados, al ser sometidos a tinción con tres bebidas cromógenas, siendo la recidiva de color de los dientes clareados, inmersos en café inmediata, en la primera inmersión. En las sucesivas inmersiones ${ }^{(20)}$, ambos grupos simultáneamente, presentan un paulatino oscurecimiento como efecto del contacto con el cromógeno, los inmersos en té, también es inmediata en la primera inmersión, posteriormente se mantienen ambos grupos estables hasta la inmersión 10, en la que presentan un brusco oscurecimiento, decreciendo paulatinamente durante las siguientes inmersiones, como efecto del contacto con el cromógeno y los inmersos en vino, del mismo modo es inmediata en la primera inmersión. Ambos grupos, presentan un oscurecimiento significativo, posterior a cada inmersión hasta la inmersión 15, después de lo cual, el oscurecimiento se estabiliza sin diferencias significativas hasta la inmersión 20.

Con respecto a las tres bebidas cromógenas utilizadas, en dientes clareados, la que causa mayor recidiva del color en el tiempo, es el vino, del mismo modo es la que causa mayor tinción en los dientes no tratados.

\section{CONFLICTOS DE INTERÉS}

Los autores declaran no tener conflictos de interés.

\section{REFERENCIAS BIBLIOGRÁFICAS}

1. Kina S, Bruguera A. Invisible: Restauraciones estéticas cerámicas. Editora Artes Médicas Ltda., 2008.

2. Nathoo SA. The chemistry and mechanisms of extrinsic and intrinsic discoloration. J Am Dent Assoc, 1997; Suppl. 128: 6-10.

3. Moncada G, Aránguiz V, Urzúa I. Blanqueamiento en odontología: Diagnóstico, mecanismos de acción, bioseguridad, consideraciones clínicas. Primera Edición. Editorial Arancibia Hnos. y Cía. Ltda., 2000.
4. Saavedra MC. Aclaramiento o blanqueamiento dental. 1999. Disponible en: http://blanqueamientodental.com/aclaramiento\%20dental.html 5. Rocha MA, Espías AF, Sánchez LA. Efecto de la peroxidasa y catalasa sobre la adhesión de brackets ortodónticos en esmalte bovino postblanqueado con peróxido de hidrógeno y peróxido de carbamida: Estudio microtensional in vitro. Dentum, 2005; 5(1): 21-28.

6. Gallagher A, Maggio B, Bowman J, Borden L, Mason S, Felix H. Clinical 
study to compare two in-office (chairside) whitening systems. The Journal of Clinical Dentistry, 2002; 13(6): 219-224.

7. Hanks CT, Fot JC, Wataha JC, Corcoran JF. Citotoxicity and dentin permeability of carbamide peroxide and hydrogen peroxide vital bleaching materials, in vitro. Journal of Dental Research, 1993; 72(5): 931-938.

8. Berger S, Coelho A, Oliveira V, Cavalli V, Giannini M. Enamel susceptibility to red wine staining after $35 \%$ hydrogen peroxide bleaching. J Appl Oral Sci, 2008; 16(3): 201-204.

9. Chng HK, Ramli HN, Yap AUJ, Lim CT. Effect of hydrogen peroxide on intertubular dentine. Journal of Dentistry, 2005; 33: 363-369.

10. Franco Pinto C, de Oliveira R, Cavalli V, Giannini M. Peroxide bleaching agent effects on enamel surface microhardness, roughness and morphology. Braz Oral Res, 2004; 18(4): 306-311.

11. Bistey T, Nagy I, Simó A, Hegedus C. In vitro FT-IR study of the effects of hydrogen peroxide on superficial tooth enamel. Journal of Dentistry, 2006; Vol. 1048

12. Greenwall L. Técnicas de blanqueamiento en odontología restauradora. Guía ilustrada. Editorial Ars Medica. $3^{\text {a }}$ Edición, 2002. España.

13. Joiner A. The bleaching of teeth: A review of the literature. Journal of Dentistry, 2006; 34(7): 412-419.

14. Heymann HO. Tooth whitening: Facts and fallacies. Br Dent J, 2005; 198(8): 514.

15. Cadenaro M, Breschi L, Antoniolli F, Mazzoni A, Di Lenarda R. Influence of whitening on the degree of conversion of dental adhesives on dentin. Eur J Oral Sci, 2006; 114(3): 257-262.

16. McKay D, Blumberg I. Review: The role of tea in human health: An update. Journal of the American College of Nutrition, 2002; 21(1): 1-13.

17. Wu C, Wei G. Tea as a functional food for oral health. Nutrition, 2002; 18(5): 443-444.

18. Gutiérrez A. Café, antioxidantes y protección a la salud. Medisan, 2002; 6(4): 72-81.

19. Nardini M, Cirillo E, Natella F, Scaccini C. Absorption of phenolic acids in human alter coffee consumption. J Agric Food Chem, 2002; 50: 5735-5741.
20. Shi X, Dalal XS, Jain AC. Antioxidant behaviour of caffeine: Efficient scavenging of hydroxyl radicals. Food Chem Toxicol, 1991; 29: 1-6.

21. Devasagayam TP, Kamat JP, Mohan H, Kesavan PC. Caffeine as an antioxidant: Inhibition of lipoperoxidation induced by reactive oxygen species. Biochim Biophys Acta, 1996; 1282: 63-70.

22. Azam S, Hadi N, Khan NU, Hadi SM. Antioxidant and prooxidant properties of caffeine, theobromine and xanthine. Med Sci Monit, 2003 9: BR325-330.

23. Bordeu E, Scarpa J. Análisis químico del vino. Segunda edición. 2000.

24. Ribereau-Gayon P, Dubouerdieu D, Doneche B, Lonvaud A. Tratado de enología: Química del vino, estabilización para tratamientos. Primera edición. Editorial Hemisferio Sur. 2003.

25. Spiller MA. Chapter 6. The chemical components of coffee. In Caffein. Edited by Gene A. Spiller. CRC Press, 1998.

26. Ghavamnasiri M, Bidar M, Habibi A, Sadegh M. The effect of 16 percente carbamide peroxide on enamel staining susceptibility. $C D A$ Journal, Nov 2006; 34(11): 873-876.

27. Catelan A, Fraga Briso A, Hermann Sundfeld R, Coelho Goiato $M$, Henrique dos Santos P. Color stability of sealed composite resin restorative materials after ultraviolet artificial aging and inmersión in staining solutions. The Journal of Prosthetic Dentistry, Apr 2011; 105(4): 236-241.

28. Toksoy F, Sahinkesen G, Yamanel K, Erdemir U, Oktay E, Ersahan $\mathrm{S}$. Influence of different drinks on the color stability of dental resin composites. European Journal of Dentistry, January 2009; 3: 51-56.

29. Yuan $J$ et al. Defining a natural tooth color space based on a 3-dimensional shade system. The Journal of Prosthetic Dentistry, 2007; 98Issue 2: 110-119.

30. Leard A, Addy M. The propensity of different brands os tea and coffee to cause staining associates with chlorhexidine. J Clinic Period, 1997; 24: $115-118$ 